\title{
Influence of Electric Field and Carrier Localization on Carrier Dynamics in AlGaN Quantum Wells
}

\author{
J. Mickevičıus ${ }^{a}$, G. TAmulaitis ${ }^{a}$, E. Kuokštis $^{a}$, M.S. Shur $^{b}$, \\ J. YANG ${ }^{c}$ AND R. GASKA ${ }^{c}$ \\ ${ }^{a}$ Institute of Materials Science and Applied Research, Vilnius University \\ Saulètekio al. 9-III, LT-10222, Vilnius, Lithuania \\ ${ }^{b}$ Department of ECE and CIE, Rensselaer Polytechnic Institute \\ Troy, NY 12180, USA \\ ${ }^{c}$ Sensor Electronic Technology, Inc. \\ 1195 Atlas Road, Columbia, SC 29209, USA
}

\begin{abstract}
Dynamics of nonequilibrium carriers in high-Al-content AlGaN/AlGaN multiple quantum wells was studied. A set of multiple quantum wells with well widths varying from 1.65 to $5.0 \mathrm{~nm}$ was grown by metal-organic chemical vapor deposition. The structures were investigated by photoluminescence spectroscopy under quasi-steady-state conditions. The observed blueshift of the photoluminescence band peak was attributed to the screening of the built-in electric field. The integrated photoluminescence intensity dependence on excitation and temperature showed a strong influence of carrier localization.
\end{abstract}

PACS numbers: 78.55.Cr, 78.67.De

\section{Introduction}

High-efficiency deep ultraviolet (UV) optoelectronic devices have a wide range of potential applications such as water and air purification, and applications in medicine and biochemistry. Deep UV emitters use high-Al-content AlGaN heterostructures providing emission at the wavelength down to $200 \mathrm{~nm}$. LEDs operating at $247 \mathrm{~nm}$ [1], $231 \mathrm{~nm}$ [2] and $227 \mathrm{~nm}$ [3] have been demonstrated. The shortest operating wavelength of $210 \mathrm{~nm}$ has been achieved in AlN-based LED [4]. However, the external quantum efficiency of the demonstrated LEDs is still very low and needs to be improved. To improve the performance of the AlGaN-based optoelectronic devices, carrier dynamics in AlGaN quantum wells (QWs) has to 
be understood in more detail. In this work, we investigate carrier dynamics in a set of high-aluminum-content $\mathrm{AlGaN} / \mathrm{AlGaN}$ multiple quantum wells (MQWs) with different thicknesses grown at otherwise identical conditions.

\section{Experimental}

The AlGaN MQWs used in this work were grown on sapphire substrate with a $1.2 \mu \mathrm{m}$ thick buffer layer of $n-\mathrm{Al}_{0.55} \mathrm{Ga}_{0.45} \mathrm{~N}$ by metal-organic chemical vapor deposition. All five $\mathrm{Al}_{x} \mathrm{Ga}_{1-x} \mathrm{~N}$ structures had ten periods consisting of barriers with aluminum content of $x=0.49$ and the same thickness of $11.5 \mathrm{~nm}$ and wells with $x=0.35$. The well thickness in different samples ranged from 1.65 to $5.0 \mathrm{~nm}$.

The samples were investigated by using photoluminescence (PL) spectroscopy under quasi-steady-state conditions. Temperature- and excitation-dependent measurements were performed. The MQW structures were excited by the 4 th harmonic $(266 \mathrm{~nm})$ of the $Q$-switched YAG:Nd laser radiation (pulse duration $4 \mathrm{~ns}$ ). The sample emission was dispersed by Jobin Yvon double monochromator HRD-1 and analyzed by an UV-enhanced PMT. A closed-cycle helium cryosystem provided temperature variation in the range from $8 \mathrm{~K}$ to $300 \mathrm{~K}$.

\section{Results and discussion}

The room temperature PL spectra measured at different excitation power densities are demonstrated in Fig. 1. The results for two AlGaN/AlGaN MQWs structures are shown for comparison: the structures with $5.0 \mathrm{~nm}$ width quantum wells (Fig. 1a) and QWs with $2.5 \mathrm{~nm}$ width wells (Fig. 1b). The excitation power density in the measurements was varied from $0.5 \mathrm{~kW} / \mathrm{cm}^{2}$ to $2 \mathrm{MW} / \mathrm{cm}^{2}$.

The PL spectra contain the band, which is caused by optical transitions between the lowest states of quantum-confined electron and holes in AlGaN quantum well. The width of the PL band is approximately $140 \mathrm{meV}$. Such a large band width indicates a large band gap modulation due to fluctuations in well width or/and in aluminum content.

With increasing excitation power density, no new bands appear in the spectra. This is an indication that no new recombination mechanisms emerge. The PL band peak position shifts to higher energies (shown by the solid line in Fig. 1a) for the structure with wide QWs of $5.0 \mathrm{~nm}$ width. However, no such shift is observed in the structure with narrow QWs of 2.5 and $1.6 \mathrm{~nm}$ width. The peak position dependences on the excitation power density are shown in Fig. 2 for the same two samples as well as for the sample with $4.1 \mathrm{~nm}$ width quantum wells. It is clearly observed that the peak blueshift is larger in wider quantum wells, whereas it becomes negligible in narrow QWs. The band blueshift with increasing excitation power density might have two reasons: filling of localized states or screening of the built-in electric field due to spontaneous piezoelectric polarization in the heterostructure. However, the negligible peak shift in the narrowest QWs points out to the predominance of the screening of the built-in electric field. The built-in 


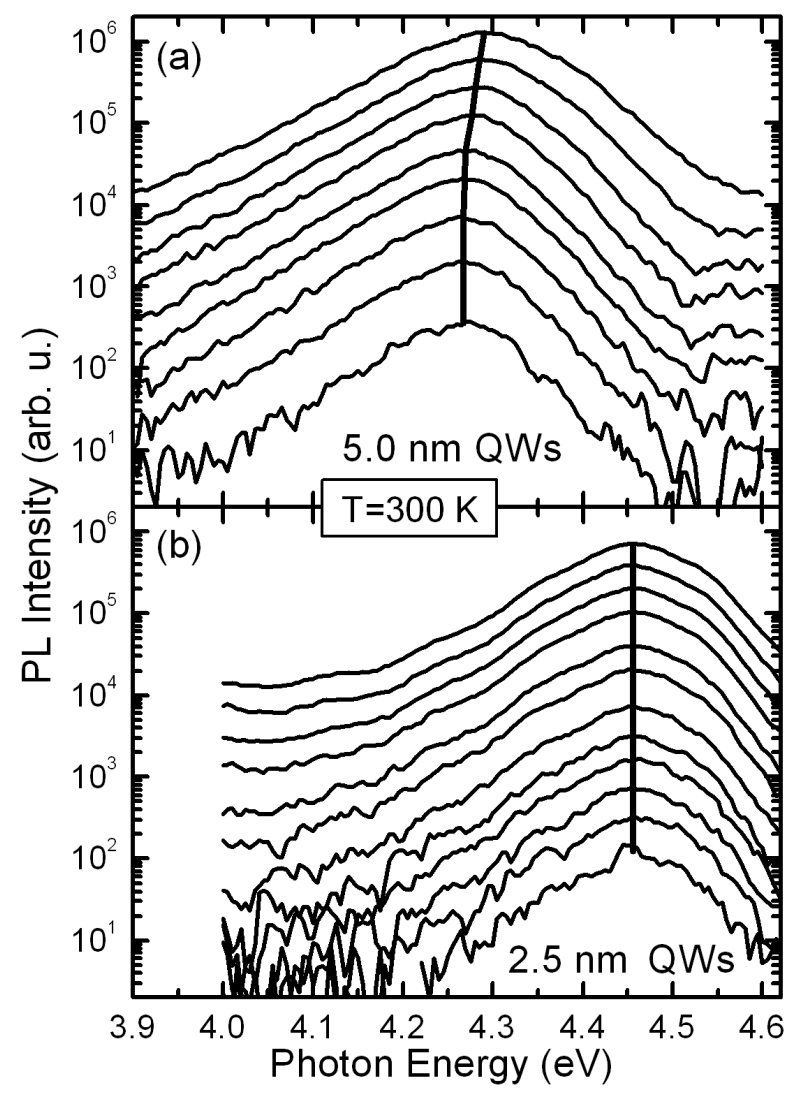

Fig. 1. PL spectra measured at different excitation power densities from $0.5 \mathrm{~kW} / \mathrm{cm}^{2}$ to $2 \mathrm{MW} / \mathrm{cm}^{2}$ for two AlGaN/AlGaN MQWs structures with well width of $5.0 \mathrm{~nm}$ (a) and $2.5 \mathrm{~nm}$ (b). All the measurements were performed at room temperature. Solid lines indicate peak position on each spectrum.

electric field results in tilting of the bottom of a quantum well and in a redshift of the energy levels of the quantum-confined electrons and holes. The shift is more pronounced in wide QWs but might be negligible in narrow ones, like in the narrowest QW under our study.

The excitation-dependent PL spectra were measured at different temperatures. Figure 3 shows the spectrally integrated PL intensity dependence at $8 \mathrm{~K}$ and $300 \mathrm{~K}$. There is a substantial difference between the dependences obtained at different temperatures. At $300 \mathrm{~K}$, the PL intensity increases nearly linearly with excitation in the entire range of pump power densities $\left(0.5 \mathrm{~kW} / \mathrm{cm}^{2}\right.$ to $\left.2 \mathrm{MW} / \mathrm{cm}^{2}\right)$. Meanwhile at $8 \mathrm{~K}$, an increase in the excitation power density above $\approx 10 \mathrm{~kW} / \mathrm{cm}^{2}$ results in a sublinear dependence.

The observed PL intensity dependences on the excitation power density can be explained by competition of the carrier recombination through radiative and 


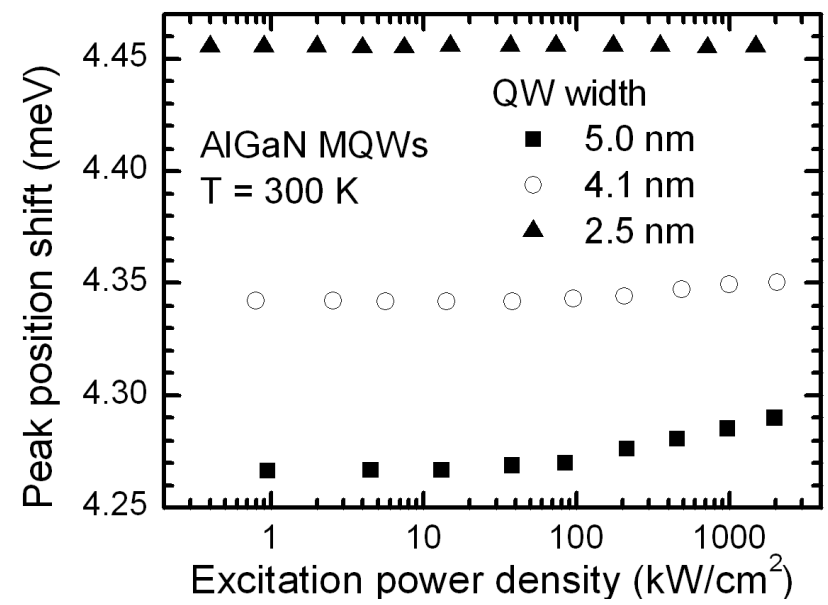

Fig. 2. PL peak position dependence on excitation power density for three AlGaN/AlGaN MQWs structures with different well widths (indicated) extracted from the PL spectra measured at room temperature.

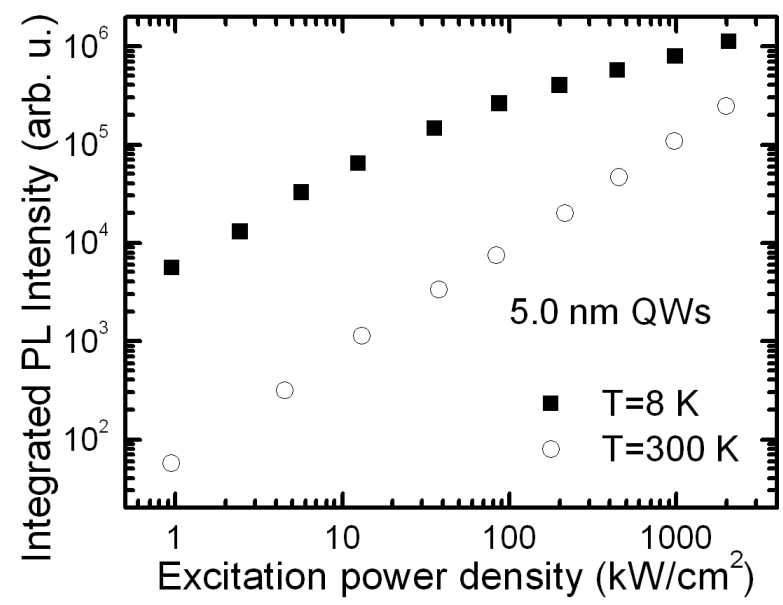

Fig. 3. Integrated PL intensity dependences on excitation power density at two different temperatures (indicated) for $\mathrm{AlGaN} / \mathrm{AlGaN}$ MQWs structure with $5.0 \mathrm{~nm}$ width wells.

nonradiative channels and by the carrier localization. At low temperatures and low excitations, all the carriers (excitons) are localized and cannot reach nonradiative recombination centers (NRCs). At moderate excitation, the carriers still fill localized states and recombine mostly radiatively, thus the pump power density dependence of the luminescence intensity is linear. At elevated excitation levels, however, most of the localized states are filled and a considerable fraction of the carriers becomes free. This makes it easier for nonequilibrium carriers to reach NRCs and recombine nonradiatively. With further increase in excitation, the frac- 
tion of the free carriers increases, and nonradiative recombination starts to play an important role. Since a larger fraction of the carriers recombine nonradiatively, the PL intensity dependence on excitation is weaker (becomes sublinear).

At elevated temperatures (e.g. at $300 \mathrm{~K}$ ), a substantial part of the carriers is already free due to thermal activation, even at the lowest excitation applied in our experiments. Thus, nonradiative recombination is a dominant recombination channel. Let us note that the PL intensity at room temperature and low excitation is by two orders of magnitude lower than that at $8 \mathrm{~K}$. This strongly supports the assumption of considerable influence of nonradiative recombination at room temperature. Meanwhile, the linear dependence of PL intensity on the pump power density indicates that the luminescence is caused by localized carriers (excitons).

The origin of carrier localization might be fluctuations in aluminum content. However, the localization is most probably caused by the well width fluctuations. Indeed, according to a simple rectangular QW model, variation of well width by one monolayer gives a difference in energy of optical transition of $7 \mathrm{meV}$ for the $5.0 \mathrm{~nm}$ width well and of $47 \mathrm{meV}$ for the $1.65 \mathrm{~nm}$ width well. The model of strong localization is also consistent with the unusual carrier lifetime dependence on QW width measured in the same samples and reported in Ref. [5], where longer carrier lifetimes were observed in narrower QWs. Such behavior was attributed to stronger localization.

\section{Conclusions}

In conclusion, a set of AlGaN/AlGaN MQWs structures with the well width varying from 1.65 to $5.0 \mathrm{~nm}$ was investigated by using temperature- and excitation-dependent photoluminescence. It was shown that at low temperatures most of carriers are in localized states and recombine predominantly in a radiative way. An increase in excitation power density results in an increase in free carrier concentration, and the free carriers are able to migrate to nonradiative recombination centers. At room temperature, the carrier recombination is strongly dominated by a nonradiative mechanism, and the light emission occurs due to linear recombination of localized carriers (excitons). An increase in the excitation power density results in a higher density of free carriers and, consequently, in screening of the built-in electric field. The decrease in the electric field in the well transforms the well from a triangular to a rectangular shape and blueshifts the PL band. This effect is more pronounced in wide QWs, while carrier localization is stronger in narrower QWs. Thus both localization and electric field play a significant role in the carrier dynamics in AlGaN QWs, and the contributions of these effects can be distinguished by studying the PL intensity and peak position dependence on excitation power density in a wide temperature range. 


\section{Acknowledgments}

The work at RPI was partially supported by the National Science Foundation under the auspices of the I/UCRC "Connection One".

\section{References}

[1] J. Deng, Y. Bilenko, A. Lunev, X. Hu, T.M. Katona, J. Zhang, M.S. Shur, R. Gaska, Jpn. J. Appl. Phys., Part 2 46, L263 (2007).

[2] H. Hirayama, T. Yatabe, N. Noguchi, T. Ohashi, N. Kamata, Appl. Phys. Lett. 91, 071901 (2007).

[3] H. Hirayama, N. Noguchi, T. Yatabe, N. Kamata, Appl. Phys. Expr. 1, 051101 (2008).

[4] Y. Taniyasu, M. Kasu, T. Makimoto, Nature 441, 325 (2006).

[5] J. Mickevičius, G. Tamulaitis, E. Kuokštis, K. Liu, M.S. Shur, J.P. Zhang, R. Gaska, Appl. Phys. Lett. 90, 131907 (2007). 